Pacific Journal of Mathematics

LATTICES WITH NO INTERVAL HOMOMORPHISMS 


\section{LATTICES WITH NO INTERVAL HOMOMORPHISMS}

\section{J. D. LAwSON}

This paper arose from the following analogous questions: (1) Does a distributive topological lattice on a continuum admit sufficiently many continuous lattice homomorphisms onto the unit interval to separate points, and (2) does a topological semilattice on a continuum admit sufficiently many continuous semilattice homomorphisms onto the unit interval to separate points? Earlier investigations of topological lattices and semilattices have provided partial positive solutions. However, examples of an infinite-dimensional distributive lattice and a one-dimensional semilattice which admit only trivial homomorphisms into the interval are presented in this paper.

A topological lattice consists of a Hausdorff space $L$ together with a pair of continuous lattice operations $\wedge, \vee: L \times L \rightarrow L$. A topological semilattice consists of a Hausdorff space $S$ together with a continuous semilattice operation $\wedge: S \times S \rightarrow S$. In the theory of topological lattices and semilattices, the following problem, raised by Dyer and Shields in [8], has received considerable attention: Does a distributive topological lattice (a topological semilattice) on a continuum admit sufficiently many continuous lattice (semilattice) homomorphisms onto the unit interval $[0,1]$ to separate points?

Anderson [2] has given an affirmative answer for finite-dimensional lattices; Davies [7] and Strauss [12] have made further contributions to the problem for the lattice case. The semilattice question has been answered affirmatively for finite-dimensional semilattices on Peano continua [11]. The purpose of this paper is to provide examples that show the answer is not yes in general. We give examples of an infinite-dimensional distributive lattice and a one-dimensional semilattice which admit only trivial homomorphisms into the interval.

Since the idempotents of an abelian topological semigroup form a semilattice, these examples have ramifications with regard to representations of such semigroups. In particular, Brown and Friedberg [6] have a range space for representations (or semicharacters) of a special class of compact abelian semigroups. These representations separate points if and only if the homomorphisms of the idempotents into the interval separate points.

1. Preliminaries. Let $S$ be a (lower) semilattice. If $A \subset S$, we define

$$
L(A)=\{y \in S: y \leqq x \text { for some } x \in A\}
$$


and

$$
M(A)=\{z \in S: x \leqq z \text { for some } x \in A\} .
$$

The set $A$ is an ideal if $L(A)=A$. The set $A$ is convex if $x, z \in A$ and $x \leqq y \leqq z$ imply $y \in A$. The following theorem is a slight modification of a theorem of Borrego [3]. It will simplify somewhat showing multiplication is continuous at a later stage.

THEOREM 1.1. Let $S$ be a compact, Hausdorff space which is algebraically a semilattice. If the graph of the partial order is closed and the operation $\wedge$ is continuous at all points of the graph, then $S$ is a topological semilattice.

Proof. Let $\left\{x_{\alpha}\right\}$ and $\left\{y_{\beta}\right\}$ be nets converging to $x$ and $y$ respectively. Let $z$ be a cluster point of the net $\left\{x_{\alpha} \wedge y_{\beta}\right\}$. Since $\left(x_{\alpha} \wedge y_{\beta}, x_{\alpha}\right)$ clusters to $(z, x)$, we conclude that $z \leqq x$. Similarly we conclude $z \leqq y$; hence $z \leqq x \wedge y$. By continuity of $\wedge$ on the graph, we conclude that $x_{\alpha} \wedge(x \wedge y)$ converges to $x \wedge y, y_{\beta} \wedge(x \wedge y)$ converges to $x \wedge y$, and hence $\left(x_{\alpha} \wedge y_{\beta}\right) \wedge x \wedge y$ converges to $x \wedge y$. Thus $x \wedge y \leqq z$ since $x_{\alpha} \wedge y_{\beta}$ clusters to $z$. Hence $z=x \wedge y$. Since $x \wedge y$ is the only cluster point, multiplication is continuous.

The next theorem is an unpublished result of D. R. Brown although apparently other researchers in the area of topological lattices and semilattices were aware of it independently.

Theorem 1.2. Let $S$ be a compact topological semilattice. Then the space $S^{\prime}$ of all closed ideals, ordered by inclusion, is a compact distributive topological lattice. The mapping sending $s$ into $L(s)$ is a topological isomorphism from $S$ into $S^{\prime}$. If $S$ is connected (metrizable), then $S^{\prime}$ is connected (metrizable).

Proof. The space $S^{\prime}$ of closed ideals is known to be a compact topological semigroup with respect to the operation

$$
A \cdot B=\{a \wedge b: a \in A, b \in B\}
$$

[9, A-7.2]. Since $A$ and $B$ are ideals, $A \cdot B \subset A \cap B \subset(A \cap B)^{2} \subset A \cdot B$. Hence $A \cdot B=A \cap B$. The union of two closed ideals is another such, and a straightforward argument shows that this operation is continuous. Hence $S^{\prime}$ is a compact distributive topological lattice.

The mapping $G$ sending $s$ into $L(s)$ is an isomorphism since

$$
L(s \wedge t)=L(s) \cap L(t) .
$$

By continuity of multiplication on the space of all closed subsets, if 
a net $s_{\alpha}$ converges to $s$, then $S \wedge s_{\alpha}$ converges to $S \wedge s$. Since $L(s)=$ $S \wedge s$, the mapping $G$ is continuous and hence a homeomorphism.

If $S$ is connected, then $S^{\prime}$ is connected since as a compact lattice it is generated by $G(S)$. If $S$ is metric, it is well-known that the space of closed subsets is metrizable; hence the subset $S^{\prime}$ is metrizable.

We now define a series which will be employed in the definition of the first example. For each positive integer $n$ larger than 1, we set

$$
\alpha_{n}=1 / m 2^{m-1} \text { where } 2^{m-1}<n \leqq 2^{m} .
$$

This series may be thought of as dividing the $m$-th term of the harmonic series into $2^{m-1}$ equal parts. Hence this series is divergent.

Proposition 1.3. For any $\varepsilon>0$, there exists a positive integer $P$ such that if $k \geqq P$, then $\sum_{n=2}^{k} \alpha_{n}+\varepsilon>\sum_{n=2}^{2 k} \alpha_{n}$.

Proof. We first note that $\sum_{n \in A} \alpha_{n}=1 / m$ if $A=\left\{n: 2^{m-1}<n \leqq 2^{m}\right\}$. Choose $q$ and $P$ such that $2 / \varepsilon<q$ and $2^{q-1}<P$. If $k \geqq P$, there exists an unique $m$ such that $2^{m-1}<k \leqq 2^{m}$. Then

$$
\sum_{n=2}^{2 k} \alpha_{n} \leqq \sum_{n=2}^{2 m+1} \alpha_{n}=\sum_{n=2}^{2 m-1} \alpha_{n}+\left(\frac{1}{m}+\frac{1}{m+1}\right) \leqq \sum_{n=2}^{k} \alpha_{n}+\frac{2}{m} .
$$

Since $m \geqq q$, we have $2 / m \leqq 2 / q<\varepsilon$; this completes the proof.

2. Examples with no interval homomorphisms. We first define some basic building blocks from which we construct our examples. Let $H^{*}$ denote $[0, \infty]$, the extended nonnegative reals; $H^{*}$ is a topological lattice with respect to its natural order. For each positive integer $i$, let $s(i)$ be the least integer with the property that $i \leqq \sum_{n=2}^{s(i)} \alpha_{n}$; such an integer exists since $\sum \alpha_{n}$ is divergent. We set $S_{i}=\prod_{n=1}^{s(i)}$ $\{0,1\}$; each $S_{i}$ is a finite lattice with respect to the coordinatewise order with $0<1$.

For $x \in S_{i}, \theta(x)$ will denote the number of zero entries of $x$. We define $\sigma_{i}: S_{i} \rightarrow H^{*}$ by (i) $\sigma_{i}(x)=\infty$ if $\theta(x)=0$, (ii) $\sigma_{i}(x)=i$ if $\theta(x)=1$, (iii) $\sigma_{i}(x)=0$ if $\theta(x)=s(i)$, and (iv) $\sigma_{i}(x)=i-\sum_{n=2}^{\theta(x)} \alpha_{n}$ for all other cases.

LEMMA 2.1. Each $\sigma_{i}$ is an order preserving function from $S_{i}$ into $H^{*}$. If $\tau>\varepsilon>0$ are fixed positive numbers, there exists a positive integer $Q$ such that if $i \geqq Q, x, y \in S_{i}, \sigma_{i}(x)>\tau, \sigma_{i}(y)>\tau$, then $\sigma_{i}(x \wedge y)>\tau-\varepsilon$.

Proof. That each $\sigma_{i}$ is order preserving is a straightforward consequence of its definition. 
Assume that $\tau>\varepsilon>0$. Choose the $P$ guaranteed by Proposition 1.3 which corresponds to $\varepsilon$. Choose $Q$ larger than $\tau+\sum_{n=2}^{2 P} \alpha_{n}$.

We suppose that $i \geqq Q, x, y \in S_{i}, \sigma_{i}(x)>\tau$ and $\sigma_{i}(y)>\tau$; we denote $x \wedge y$ by $z$. Either $\theta(z) \leqq 2 \theta(x)$ or $\theta(z) \leqq 2 \theta(y)$ obtains; we arbitrarily assume $\theta(z) \leqq 2 \theta(x)$ (the reason one of the inequalities prevails is that $x \wedge y$ can have at most twice as many zero entries as one of $x$ or $y$ ). We note from the definition of $\sigma_{i}$ that in all cases $\sigma_{i}(z) \geqq i-\sum_{n=2}^{\theta(z)} \alpha_{n}$ if the summation is interpreted to be 0 for $\theta(z)$ equal to 0 or 1 .

If $\theta(x) \leqq P$, then

$$
\sigma_{i}(z) \geqq i-\sum_{n=2}^{\theta(z)} \alpha_{n} \geqq Q-\sum_{n=2}^{2 \theta(x)} \alpha_{n} \geqq Q-\sum_{n=2}^{2 P} \alpha_{n} \geqq \tau ;
$$

the last inequality follows from the choice of $Q$. Hence $\sigma_{i}(z)>\tau-\varepsilon$ if $\theta(x) \leqq P$.

If $P<\theta(x)$, then

$$
\sigma_{i}(z) \geqq i-\sum_{n=2}^{\theta(z)} \alpha_{n} \geqq i-\sum_{n=2}^{2 \theta(x)} \alpha_{n} \geqq i-\left(\sum_{n=2}^{\theta(x)} \alpha_{n}+\varepsilon\right)=\sigma_{i}(x)-\varepsilon>\tau-\varepsilon .
$$

Hence $\sigma_{i}(z)>\tau-\varepsilon$ for both cases. We now define the first example. We denote $H^{*} \times \prod_{i=1}^{\infty} S_{i}$ by $K$. With coordinatewise order $K$ is a topological lattice homeomorphic to the Cartesian product of an interval and the Cantor set.

ExAmple 1. We define $L=\left\{\left(t,\left(x_{i}\right)_{i=1}^{\infty}\right) \in K: t \leqq \sigma_{i}\left(x_{i}\right)\right.$ for all $\left.i\right\}$. With respect to the order inherited from $K, L$ is a compact, onedimensional topological lattice. If $A$ is a subsemilattice of $L$ with respect to the cap operation and if $1 \in A^{\circ}$ (where 1 denotes the largest element of $L)$, then $A \cap\left(0 \times \Pi S_{i}\right) \neq \varnothing$.

Proof. (1) $L$ is compact.

Suppose $\left(t,\left(x_{i}\right)\right) \notin L$. Then $t>\sigma_{n}\left(x_{n}\right)$ for some $n$. There exists an open neighborhood $U$ of $\left(t,\left(x_{i}\right)\right)$ such that if $\left(s,\left(y_{i}\right)\right) \in U$, then $s>\sigma_{n}\left(x_{n}\right)$ and $y_{n}=x_{n}$; then $s>\sigma_{n}\left(x_{n}\right)=\sigma_{n}\left(y_{n}\right)$ implies $\left(s,\left(y_{i}\right) \notin L\right.$. Hence $K \backslash L$ is open and $L$ is compact.

(2) $L$ is algebraically a lattice.

With respect to the cup operation, $L$ is a subsemilattice of $K$. This follows from the fact each $\sigma_{i}$ is order preserving.

To complete this part, we show that if $\left(s,\left(x_{i}\right)\right),\left(t,\left(y_{i}\right)\right) \in L$, then $\left(u,\left(z_{i}\right)\right)$ is a greatest lower bound in $L$ where

$$
u=s \wedge t \wedge \inf .\left\{\sigma_{i}\left(z_{i}\right): 1 \leqq i\right\}
$$

and $z_{i}=x_{i} \wedge y_{i}$. By its definition $\left(u,\left(z_{i}\right)\right)$ is a lower bound and a member of $L$. Let $\left(r,\left(w_{i}\right)\right)$ be another lower bound for $\left(s,\left(x_{i}\right)\right)$ and $\left(t,\left(y_{i}\right)\right)$ in $L$. Then $r \leqq s \wedge t$ and $w_{i} \leqq x_{i} \wedge y_{i}=z_{i}$ for each $i$; hence $\sigma_{i}\left(w_{i}\right) \leqq \sigma_{i}\left(z_{i}\right)$ for each $i$. Since $\left(r,\left(w_{i}\right)\right) \in L$, then $r \leqq \inf \left\{\sigma_{i}\left(w_{i}\right): 1 \leqq i\right\} \leqq \inf \left\{\sigma_{i}\left(z_{i}\right): 1 \leqq i\right\}$. 
Hence $r \leqq u$; and thus $\left(u,\left(z_{i}\right)\right)$ is a $g l b$ in $L$.

(3) $L$ is a topological lattice.

The cup operation is continuous since $L$ is a subsemilattice of $K$ with respect to this operation. This implies that the partial order on $L$ has closed graph.

Let $x=\left(s,\left(x_{i}\right)\right)$ and $y=\left(t,\left(y_{i}\right)\right)$ be elements of $L$. To show continuity of multiplication, we may assume that $y \leqq x$ by Theorem 1.1.

We first consider the case that $0<t, s<\infty$. For a positive integer $N$ and $\varepsilon>0$, let $W=\left\{\left(u, z_{i}\right) \in L: t-3 \varepsilon<u<t+3 \varepsilon, y_{i}=z_{i}\right.$ for $i \leqq N\}$ be a basic neighborhood of $y$ where $3 \varepsilon<t$. Let $Q$ be the positive integer guaranteed by Lemma 2.1 for $\tau=t-\varepsilon$ and $\varepsilon$; we set $M=\max \{N, Q\}$. We define neighborhoods $U$ and $V$ of $x$ and $y$ resp. by

$U=\left\{\left(s^{\prime},\left(a_{i}\right)\right) \in L: s-\varepsilon<s^{\prime}<s+\varepsilon, a_{i}=x_{i}\right.$ for $\left.i \leqq M\right\}$ and

$V=\left\{\left(t^{\prime},\left(b_{i}\right)\right) \in L: t-\varepsilon<t^{\prime}<t+\varepsilon, b_{i}=y_{i}\right.$ for $\left.i \leqq M\right\}$.

To complete the proof, we show $U \wedge V \subset W$.

Let $\left(s^{\prime},\left(a_{i}\right)\right) \in U$ and $\left(t^{\prime},\left(b_{i}\right)\right) \in V$ and let $\left(u,\left(z_{i}\right)\right)$ be their greatest lower bound in $L$, i.e., $z_{i}=a_{i} \wedge b_{i}$ for all $i$ and

$$
u=s^{\prime} \wedge t^{\prime} \wedge \inf \left\{\sigma_{i}\left(z_{i}\right): 1 \leqq i\right\} .
$$

We have immediately $u \leqq t^{\prime}<t+3 \varepsilon$ and $z_{i}=a_{i} \wedge b_{i}=x_{i} \wedge y_{i}=y_{i}$ for $i \leqq N$ since $N \leqq M$. Since $\left(t^{\prime},\left(b_{i}\right)\right)$ is an element of $V$ and hence of $L$, we have $t-\varepsilon<t^{\prime} \leqq \sigma_{i}\left(b_{i}\right)$ for all $i$. Similarly since $t \leqq s$, we have $t-\varepsilon \leqq s-\varepsilon<s^{\prime} \leqq \sigma_{i}\left(a_{i}\right)$. If $i \leqq M$, then

$$
t-2 \varepsilon<t-\varepsilon<\sigma_{i}\left(b_{i}\right)=\sigma_{i}\left(a_{i} \wedge b_{i}\right)=\sigma_{i}\left(z_{i}\right)
$$

since $a_{i} \wedge b_{i}=x_{i} \wedge y_{i}=y_{i}=b_{i}$. If $M<i$, then $Q<i$ and

$$
(t-\varepsilon)-\varepsilon<\sigma_{i}\left(a_{i} \wedge b_{i}\right)
$$

by Lemma 2.1. Hence $t-3 \varepsilon<t-2 \varepsilon \leqq s^{\prime} \wedge t^{\prime} \wedge \inf \left\{\sigma_{i}\left(z_{i}\right): 1 \leqq i\right\}=u$. Thus $\left(u,\left(z_{i}\right)\right) \in W$.

The case $t=0$ is straightforward and omitted. The case that one or both of $t$ and $s$ are $\infty$ can be handled by a slight modification of the above argument.

(4) $L$ is one-dimensional.

This follows from the fact that $L$ is homeomorphic to a closed subset of the Cartesian product of the Cantor set and unit interval.

(5) If $A$ is a subsemilattice and $1 \in A^{\circ}$, then $A \cap\left(0 \times \Pi S_{i}\right) \neq \varnothing$. Note that $\left(\infty,\left(x_{i}\right)\right)$ where each $x_{i}$ has entries all 1 is an element of $L$, and hence is the 1 for $L$. There exists at 1 a basis of open sets of the form $U=\left\{\left(r,\left(x_{i}\right)\right) \in L: j<r, x_{i}\right.$ has entries all 1 for $\left.i \leqq j\right\}$ where $j$ is a positive integer. We assume $j$ is chosen so that $U \subset A^{\circ}$. We define $T$ to be all elements of the form $\left(j+1,\left(x_{i}\right)\right)$ such that $x_{i}$ has entries all 1 for $i \neq j+1$ and $x_{j+1}$ has one zero entry. Then $T$ has $s(j+1)$ elements. For each element of $T$, 


$$
\inf \left\{\sigma_{i}\left(x_{i}\right): 1 \leqq i\right\}=\sigma_{j+1}\left(x_{j+1}\right)=j+1 ;
$$

hence $T \subset L$ and thus $T \subset U$. Let $\left(t,\left(z_{i}\right)\right)$ be the greatest lower bound in $L$ of $T$. Since $A$ is a subsemilattice, $\left(t,\left(z_{i}\right)\right) \in A$. Since $\left(t, z_{i}\right) \in L$, $t \leqq \sigma_{j+1}\left(z_{j+1}\right)=0$ since $z_{j+1}$ has entries all zero. Hence $t=0$.

EXAmple 2. Let $I$ denote all elements of $L$ with first entry zero; $I$ is an ideal of $L$ with respect to the cap operation. The Rees quotient $S=L / I$ is a compact, connected one-dimensional metric semilattice with identity which admits no nontrivial semilattice homomorphisms into the unit interval.

Proof. It is easily verified that the set $I=\left\{\left(0,\left(x_{i}\right)\right) \in L\right\}$ is a closed ideal of $L$ with respect to the cap operation. Hence $S=L / I$, the Rees quotient, is a compact topological semilattice.

Since $S$ is topologically a subset of the cone over the Cantor set, $S$ is metric and one-dimensional. If $\left(t,\left(x_{i}\right)\right) \in L$, then $\left\{\left(r,\left(y_{i}\right)\right): r \leqq t\right.$, $y_{i}=x_{i}$ for all $\left.i\right\}$ is a connected subset of $L$ which meets $I$. Hence in $S$ each element lies in the component of 0 ; thus $S$ is connected.

Assume that there does exist a nontrivial continuous homomorphism $h$ from $S$ into [0,1]. Then $h(1)>h(0)$. If $f$ denotes the natural homomorphism from $L$ onto $S$, then $h f$ is a continuous homomorphism from $L$ into the unit interval such that $h f(I)=h(0)$. Choose $r$ such that $h(1)>r>h(0)$. Then $(h f)^{-1}[r, h(1)]$ is a neighborhood of 1 in $L$, a subsemilattice of $L$, and misses $I$. However, no subset of $L$ has these properties. Hence no nontrivial $h$ exists.

ExAmple 3. Let $S^{\prime}$ denote the set of all closed ideals of $S$, the semilattice of Example 2. Then $S^{\prime}$ is a compact connected metrizable distributive topological lattice. With respect to the cap operation, $S^{\prime}$ has no nontrivial finite-dimensional homomorphic images; hence, in particular, $S^{\prime}$ admits no nontrivial lattice homomorphisms into the unit interval.

Proof. By Theorem 1.2 $S^{\prime}$ is a compact, connected, metrizable, distributive topological lattice and the mapping $G$ from $S$ into $S^{\prime}$ sending $s$ into $L(s)$ is a topological isomorphism. Since $G(0)=0$ and $G(1)=$ 1 , $S^{\prime}$ admits no nontrivial cap homomorphisms into the unit interval, because any such composed with $G$ would be a nontrivial homomorphism from $S$ into the interval.

Suppose that $h$ is a continuous cap homomorphism from $S^{\prime}$ onto $T$, a finite-dimensional topological semilattice. Since $S^{\prime}$ is a compact, connected topological lattice, it is locally connected [1]; hence $T$ is locally connected. But then, by [11], if $T$ is nontrivial, it possesses nontrivial 
homomorphisms into the interval. The composition would be a nontrivial homomorphism from $S^{\prime}$ into the interval, and we have just seen such does not exist. Hence $T$ is trivial.

These examples shed some light on the subject of intrinsic topologies in lattices. Birkhoff [4] describes several ways a lattice may be topologized from its algebraic structure. It has been shown that in a compact topological lattice which is metrizable the topology of the lattice agrees with the order topology (see [12] or [10]). Hence Examples 1 and 3 both have the order topology.

The question has been asked whether the topology of a compact topological lattice agrees with the interval topology [10]. Strauss [12] showed that if this is true and if the lattice is distributive, then the lattice admits nontrivial continuous homomorphisms into the unit interval. Hence the lattice of Example 3 does not have the interval topology.

It is a pleasure to thank Professors D. R. Brown and R. J. Koch for their encouragement and Professors John Hildebrant and Bernard Madison for their patient listening.

\section{BIBLIOGRAPHY}

1. L. W. Anderson, On the distributivity and simple connectivity of plane topological lattices, Trans. Amer. Math. Soc. 91 (1959), 102-112.

2. - The existence of continuous lattice homomorphisms, J. London Math. Soc. 37 (1962), 60-62.

3. G. Birkhoff, Lattice theory, Amer. Math. Soc. Colloquim Publications, 3rd ed., vol XXV, Amer. Math. Soc., Providence, R. I., 1967.

4. J. T. Borrego, Continuity of the operation of a semilattice, Notices Amer. Math. Soc. 16 (1969), 171.

5. D. R. Brown, Topological semilattices on the two cell, Pacific J. Math. 15 (1965), $35-46$.

6. D. R. Brown and M. Friedberg, A new notion of semicharacters, (to appear).

7. E. B. Davies, The existence of characters on topological lattices, J. London Math. Soc. 43 (1968), 217-220.

8. E. Dyer and A. S. Shields, Connectivity of topological lattices, Pacific J. Math. 9 (1959), 443-447.

9. K. H. Hofmann and P. S. Mostert, Elements of compact semigroups, Charles E. Merill Books, Inc., Columbus, Ohio, 1966.

10. J. D. Lawson, Vietoris mappings and embeddings of topological semilattices, University of Tennessee Dissertation, 1967.

11. - Topological semilattices with small semilattices, (to appear in J. London Math. Soc.).

12. D. P. Strauss, Topological lattices, Proc. London Math. Soc. 18 (1968), 217-230.

Received April 3, 1969.

Louisiana State University

Baton Rouge, Louisiana 



\section{PACIFIC JOURNAL OF MATHEMATICS}

\section{EDITORS}

\author{
H. SAMELSON \\ Stanford University \\ Stanford, California 94305 \\ RICHARD PIERCE \\ University of Washington \\ Seattle, Washington 98105
}

J. DugundJI

Department of Mathematics University of Southern California Los Angeles, California 90007

BASIL GORDON*

University of California

Los Angeles, California 90024

\section{ASSOCIATE EDITORS}
E. F. BECKENBACH
B. H. NEUMANN
F. WOLE
K. YOSHIDA

\section{SUPPORTING INSTITUTIONS}

\author{
UNIVERSITY OF BRITISH COLUMBIA \\ CALIFORNIA INSTITUTE OF TECHNOLOGY \\ UNIVERSITY OF CALIFORNIA \\ MONTANA STATE UNIVERSITY \\ UNIVERSITY OF NEVADA \\ NEW MEXICO STATE UNIVERSITY \\ OREGON STATE UNIVERSITY \\ UNIVERSITY OF OREGON \\ OSAKA UNIVERSITY \\ UNIVERSITY OF SOUTHERN CALIFORNIA
}

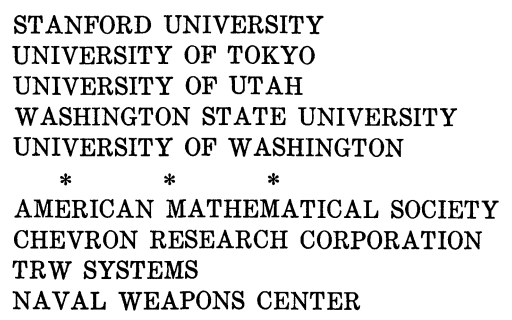

The Supporting Institutions listed above contribute to the cost of publication of this Journal, but they are not owners or publishers and have no responsibility for its content or policies.

Mathematical papers intended for publication in the Pacific Journal of Mathematics should be in typed form or offset-reproduced, (not dittoed), double spaced with large margins. Underline Greek letters in red, German in green, and script in blue. The first paragraph or two must be capable of being used separately as a synopsis of the entire paper. The editorial "we" must not be used in the synopsis, and items of the bibliography should not be cited there unless absolutely necessary, in which case they must be identified by author and Journal, rather than by item number. Manuscripts, in duplicate if possible, may be sent to any one of the four editors. Please classify according to the scheme of Math. Rev. 36, 1539-1546. All other communications to the editors should be addressed to the managing editor, Richard Arens, University of California, Los Angeles, California, 90024.

50 reprints are provided free for each article; additional copies may be obtained at cost in multiples of 50 .

The Pacific Journal of Mathematics is published monthly. Effective with Volume 16 the price per volume (3 numbers) is $\$ 8.00$; single issues, $\$ 3.00$. Special price for current issues to individual faculty members of supporting institutions and to individual members of the American Mathematical Society: $\$ 4.00$ per volume; single issues $\$ 1.50$. Back numbers are available.

Subscriptions, orders for back numbers, and changes of address should be sent to Pacific Journal of Mathematics, 103 Highland Boulevard, Berkeley, California, 94708.

PUBLISHED BY PACIFIC JOURNAL OF MATHEMATICS, A NON-PROFIT CORPORATION

Printed at Kokusai Bunken Insatsusha (International Academic Printing Co., Ltd.), 7-17, Fujimi 2-chome, Chiyoda-ku, Tokyo, Japan.

* Acting Managing Editor. 


\section{Pacific Journal of Mathematics}

\section{Vol. 32, No. $2 \quad$ February, 1970}

Harry P. Allen and Joseph Cooley Ferrar, Jordan algebras and exceptional subalgebras of the exceptional algebra $E_{6} \ldots \ldots \ldots \ldots \ldots \ldots \ldots 283$

David Wilmot Barnette and Branko Grünbaum, Preassigning the shape of a

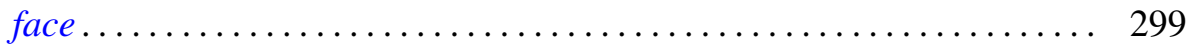

Robert Francis Craggs, Involutions of the 3-sphere which fix 2-spheres . . . . 307

David William Dean, Bor-Luh Lin and Ivan Singer, On k-shrinking and $k$-boundedly complete bases in Banach spaces ................ 323

Martin Engert, Finite dimensional translation invariant subspaces ....... 333

Kenneth Lewis Fields, On the global dimension of residue rings ......... 345

Howard Gorman, The Brandt condition and invertibility of modules ....... 351

Benjamin Rigler Halpern, A characterization of the circle and interval ..... 373

Albert Emerson Hurd, A uniqueness theorem for second order quasilinear

hyperbolic equations ............................... 415

James Frederick Hurley, Composition series in Chevalley algebras ...... 429

Meira Lavie, Disconjugacy of linear differential equations in the complex

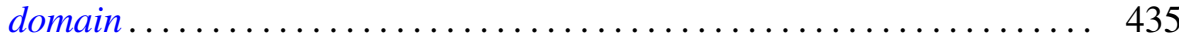

Jimmie Don Lawson, Lattices with no interval homomorphisms ......... 459

Roger McCann, A classification of center-foci ................. 467

Evelyn Rupard McMillan, On continuity conditions for functions . . . . . . . 479

Graciano de Oliveira, A conjecture and some problems on permanents .... 495

David L. Parrott and S. K. Wong, On the Higman-Sims simple group of order $44,352,000$.

Jerome L. Paul, Extending homeomorphisms ................. 517

Thomas Benny Rushing, Unknotting unions of cells .............. 521

Peter Russell, Forms of the affine line and its additive group.......... 527

Niel Shilkret, Non-Archimedean Gelfand theory ................. 541

Alfred Esperanza Tong, Diagonal submatrices of matrix maps.......... 551 